\title{
Vulnerabilidade da população negra brasileira frente a evolução da pandemia por COVID-19
}

Vulnerability of the Black Population in Brazil to the Evolution of the COVID-19 Pandemic

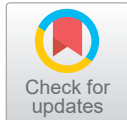

Vulnerabilidad de la población negra brasileña ante la evolución de la pandemia por COVID-19

Como citar este artigo:

Ferreira, Ricardo Bruno Santos; Camargo, Climene Laura de. Vulnerabilidade da população negra brasileira frente à evolução da pandemia por COVID-19. Revista Cuidarte. 2021;12(2):e1322. http://dx.doi.org/10.15649/cuidarte.1322

Revista Cuidarte

Rev Cuid. May - Ago 2021; 12(2): e1322

doij http://dx.doi.org/10.15649/cuidarte.1322

E-ISSN: 2346-3414

(1) Ricardo Bruno Santos Ferreira ${ }^{1}$

(1) Climene Laura de Camargo²

1 Universidade do Estado da Bahia - UNEB DEDC XII- Guanambi (BA). Brasil. Enfermeiro assistencial no Hospital do Rim de Guanambi. Bahia. Brasil. Autor correspondente. E-mail: ricardobrunoenf@gmail.com

2 Professora da Universidade Federal da Bahia - UFBA - Salvador (BA). Brasil. E-mail:

climenecamargo@hotmail.com

\section{Resumo}

Introdução: Devido as iniquidades históricas, o contexto pandêmico do COVID-19 tem impactado de maneira distinta os segmentos sociais, exigindo um acompanhamento especial às minorias étnico-raciais que vivem em situação de vulnerabilidade. Nesse sentido, o estudo tem como objetivo analisar a vulnerabilidade da população negra brasileira frente à evolução da pandemia por COVID-19. Materiais e Métodos: Trata-se de um estudo documental, descritivo, quantitativo, realizada a partir dos boletins epidemiológicos coronavírus publicados pelo Ministério da Saúde do Brasil. Foram avaliadas duas variáveis dos boletins epidemiológicos coronavírus a partir da raça/cor: hospitalizações por síndrome respiratória aguda grave e óbito por Covid-19. Resultados: Entre os boletins no 9 e n० 18 evidenciouse o aumento sustentado nas taxas de internação por síndrome respiratória aguda grave (de $23,9 \%$ para $54,7 \%$ ) e óbito (de $34,3 \%$ para $61,3 \%$ ) entre pessoas de raça/cor negra e redução constante na hospitalização (de $73 \%$ para $43,3 \%$ ) e óbito (de $62,9 \%$ para $36,5 \%$ ) entre pessoas brancas. Constata-se que pessoas negras têm maiores chances de internação por SRAG e morte por COVID-19 no Brasil, o que implica maior situação de vulnerabilidade. Discussão: Acredita-se que esse cenário desfavorável entre pessoas negras se deve a dificuldade ou impossibilidade de realização de isolamento social, à prevalência de comorbidades que precipitam quadros graves do COVID-19, as dificuldades no acesso aos serviços de saúde e ao racismo institucional. Conclusão: faz-se necessário a implementação de uma rede de proteção social a esse grupo racial, com o objetivo de reduzir o acometimento da doença e a letalidade do vírus.

Palavras chave: Betacoronavirus; Grupo com Ancestrais do Continente Africano; Racismo.

Recebido: junho 20 de 2020

Aceito: fevereiro 2 de 2021

Publicado: maio 18 de 2021 $\square *$ Correspondencia

Ricardo Bruno Santos Ferreira

E-mail: ricardobrunoenf@gmail.com 


\title{
Vulnerability of the Black Population in Brazil to the Evolution of the COVID-19 Pandemic
}

\begin{abstract}
Introduction: Due to the historical inequality, the COVID-19 pandemic has impacted social groups differently, which calls for special assistance to ethnic-racial minorities living in a situation of vulnerability. In light of this, this study is aimed to analyze the vulnerability of the black population in Brazil to the evolution of the COVID-19 pandemic. Materials and Methods: A quantitative descriptive study following a documentary method was conducted based on the coronavirus disease epidemiological bulletins published by the Brazilian Ministry of Health. Two variables were assessed from epidemiological bulletins regarding race/color: hospitalizations for severe acute respiratory syndrome and deaths due to COVID-19. Results: A steady increase in rates of hospitalization for severe acute respiratory syndrome (from $23.9 \%$ to $54.7 \%$ ) and death (from $34.3 \%$ to $61.3 \%$ ) was observed among the black population in bulletins $9-18$, contrary to the steady decrease in rates of hospitalization (from $73 \%$ to $43.3 \%$ ) and death (from $6.29 \%$ to $36.5 \%$ ) among the white people. It was also found that black people are more likely to be hospitalized for SARS infection and death due to COVID-19 in Brazil, suggesting that they are at a higher vulnerability. Discussion: This unfavorable scenario for the black population is considered to arise from the difficulty or impossibility of social integration, the prevalence of comorbidities that trigger severe COVID-19 forms, difficulties in accessing healthcare services and institutional racism. Conclusion: The implementation of a social protection network is necessary to reduce the disease infection and the mortality of the virus.
\end{abstract}

Key words: Betacoronavirus; African Continental Ancestry Group; Racism.

\section{Vulnerabilidad de la población negra brasileña ante la evolución de la pandemia por COVID-19}

\section{Resumen}

Introducción: Debido a la inequidad histórica existente, la pandemia del COVID-19 ha impactado a los grupos sociales de forma diferente, lo que exige un acompañamiento especial a las minorías étnicoraciales que viven en situación de vulnerabilidad. En este sentido, el presente estudio tiene como objetivo analizar la vulnerabilidad de la población negra brasileña frente a la evolución de la pandemia por COVID-19. Materiales y métodos: Se realizó un estudio documental descriptivo de enfoque cuantitativo a partir de los boletines epidemiológicos sobre coronavirus publicados por el Ministerio de Salud de Brasil. Se evaluaron dos variables en los boletines epidemiológicos con base en la raza/color: hospitalizaciones por síndrome respiratorio agudo grave y muertes por COVID-19. Resultados: Entre los boletines 9 y 18 se evidenció un aumento sostenido de las tasas de hospitalización por síndrome respiratorio agudo grave (del $23.9 \%$ al $54.7 \%$ ) y de muerte (del $34.3 \%$ al $61.3 \%$ ) entre la población negra, así como una reducción constante de la tasa de hospitalización (del 73\% al $43.3 \%$ ) y de muerte (del $62.9 \%$ al $36.5 \%$ ) entre la población blanca. Se observó que las personas de raza negra tienen una mayor probabilidad de hospitalización por infección por SARS y muerte por COVID-19 en Brasil, lo que supone que se encuentran en una mayor situación de vulnerabilidad. Discusión: Se considera que este escenario desfavorable para las personas de raza negra se debe a la dificultad o imposibilidad de integración social, la prevalencia de comorbilidades que desencadenan cuadros graves de COVID-19, las dificultades de acceso a los servicios de salud y el racismo institucional. Conclusión: Se hace necesaria la implementación de una red de protección social para este grupo racial a fin de reducir el contagio de la enfermedad y la letalidad del virus.

Palabras clave: Betacoronavirus. Grupo de Ascendencia Continental Africana. Racismo. 


\section{Introdução}

O Sars-cov-2 ou Covid-19, recentemente descoberto no continente Asiático, é um vírus zoonótico que causa infecção do trato respiratório. Em pouco tempo, o vírus se disseminou por todos os continentes, adquiriu status de pandemia e gerou uma das maiores crises sanitárias que o mundo moderno presenciou'.

No Brasil, até o dia 18 de junho de 2020 foram confirmados 978.142 casos e 47.748 óbitos, o que coloca o país em segundo lugar em número de óbitos e casos confirmados no mundo, atrás apenas dos Estados Unidos. Além disso, entre o período de 19 de maio a 18 de junho de 2020, o Brasil registrou diariamente uma média de 998,5 mortes e 23,3 mil novos casos, o que coloca o país como um dos maiores epicentros da doença no mundo. A alta incidência, somada a subnotificação devido ao baixo número de testes realizados auxilia o país a manter a taxa de letalidade em $4,9 \%$, uma das maiores do mundo 2 .

O aumento significativo do número de novos casos de coronavírus implica na necessidade de políticas específicas para os grupos minoritários que vivem situação de vulnerabilidade e historicamente apresentaram piores desfechos no processo saúdedoença, como a população negra.

Sabe-se que a maioria das pessoas com Covid-19
O aumento significativo do número de novos casos de coronavírus implica na necessidade de políticas específicas para os grupos minoritários que vivem situação de vulnerabilidade e historicamente apresentaram piores desfechos no processo saúde-doença, como a população negra.

são assintomáticas ou desenvolvem quadros leves ou moderados da doença. Segundo a Organização Mundial de Saúde (OMS) e o Ministério da Saúde (MS) do Brasil, os casos graves, normalmente, estão associados à idade ou presença de outra comorbidade como diabetes mellitus (DM), hipertensão arterial sistêmica (HAS), doença pulmonar crônica, imunossupressão ou obesidade ${ }^{1,2}$.

Nota-se que a variável raça-cor não foi considerada pela OMS e MS brasileiro como um fator de risco para o acometimento ou evolução para o quadro mais grave da doença. Acrescenta-se ainda que a Fundação Oswaldo Cruz (FIOCRUZ), através do observatório COVID-19, emitiu uma nota técnica intitulada COVID-19 E VULNERBILIDADES - CONSIDERAÇÕES SOBRE PROTEÇÃO SOCIAL NAS FAVELAS ${ }^{3}$, esta, porém, não faz nenhuma consideração acerca da vulnerabilidade imposta à população negra.

Essa invisibilidade da população negra também pode ser evidenciada nos estudos produzidos até o momento nas bases de dados internacionais. Ao realizar uma busca na Medical Literature Analysis and Retrieval System Online (Medline) e Scientific Electronic Library Online (Scielo) com os descritores "sars-cov-2" e "Ethnic Inequality", e, "sars-cov-2" e "african continental ancestry group" conectados com o operador booleano AND, foram encontrados apenas 15 artigos com temática relacionada, entretanto, nenhum deles considera o contexto da população negra brasileira. Os estudos encontrados discutem, sobretudo, a realidade da população dos Estados Unidos em que, assim como no Brasil, o negro possui longa história de desvantagem e iniquidade.

Nesse sentido, por tratar-se de uma doença de implicações ainda desconhecidas, de fácil contágio e com impactos sociais importantes, acredita-se que toda avaliação do contexto saúde-doença do Covid-19 deve ser guiada pelos determinantes sociais em saúde (DSS). Entendemos os DSS como fatores que influenciam diretamente na condição de saúde, dentre os quais destacamos 
os sociais, econômicos, étnico-raciais, comportamentais e psicológicos ${ }^{4}$. Ou seja, populações em situação de vulnerabilidade, como a população negra, necessitam de maior atenção das autoridades sanitárias.

Ademais, existe vasta literatura que aborda as iniquidades na saúde da população negra, desde o nascimento até a morte, mesmo quando se controla os fatores socioeconômicos. Destacamos, por exemplo, que mulheres negras têm piores condições de assistência pré-natal e ao parto ${ }^{5}$, além de que bebês pretos possuem mais chances de morrer no primeiro ano de vida, em comparação aos brancos ${ }^{6}$. Ainda, no Brasil, pessoas negras são mais acometidas por doenças orais $^{7}$, doenças falciformes e quadros que precipitam piores prognósticos do coronavírus como diabetes mellitus, hipertensão arterial e insuficiência renal crônica ${ }^{8}$, algo semelhante ao encontrado nos Estados Unidos ${ }^{9}$. Há também discrepâncias raciais no acesso ao transplante renal, apresentando maiores dificuldades para pessoas negras ${ }^{10}$.

É a partir dessa lacuna que o presente estudo revela sua importância. Nesse sentido, traçou-se como objetivo analisar a vulnerabilidade da população negra brasileira frente à evolução da pandemia por COVID-19.

\section{Materiais e Métodos}

Trata-se de um estudo documental, descritivo, exploratório, de abordagem quantitativa, desenvolvido a partir dos boletins epidemiológicos coronavírus publicados pelo Ministério da Saúde do Brasil.

Os boletins epidemiológicos são publicações eletrônicas de acesso livre, divulgadas de forma periódica, de cunho técnico-científico para os casos de monitoramento e investigação de doenças em surto como o coronavírus. Trata-se de uma ferramenta de vigilância utilizada pelo Ministério da Saúde para disseminar informações qualificadas que contribuem para construção de políticas públicas de saúde no Brasil. Dessa forma, o Ministério da Saúde utiliza os boletins como um instrumento oficial do Estado para analisar a situação epidemiológica do COVID-19.

Os dados foram levantados no período de 01 a 15 de junho de 2020. Foi realizado a leitura de todos os 18 boletins epidemiológico coronavírus publicados pelo Ministério da Saúde para selecionar os boletins que atendiam aos critérios de elegibilidade. Foram avaliadas duas variáveis dos boletins epidemiológicos coronavírus a partir da raça/cor: hospitalizações por síndrome respiratória aguda grave (SRAG) e óbito por Covid-19. Foram incluídos no estudo todos os boletins epidemiológicos coronavírus que apresentaram essas variáveis estratificadas por raça/cor, publicadas no site oficial do Ministério da Saúde até o dia 20 de junho de 2020. Nesse sentido, foram avaliados os boletins epidemiológicos $n^{\circ} 09, n^{\circ} 10, n^{\circ} 11, n^{\circ} 13, n^{\circ} 14, n^{\circ}$ $15, n^{\circ} 16, n^{\circ} 17$ e no $18^{11-19}$.

Os dados foram organizados em uma planilha no Microsoft Excel ${ }^{\circledR}$ a partir da raça/cor autodeclarada: branca, negra (pretos e pardos), amarela ou indígena e analisados através de frequências. Os dados ignorados ou sem informação não foram utilizados para análise.

Por tratar-se de informações de domínio público e não identificar pessoas, o estudo não necessitou ser submetido para avaliação do Comitê de Ética e Pesquisa. 


\section{Resultados}

O gráfico 1 mostra a evolução da taxa de hospitalização por Síndrome Respiratória Aguda Grave (SRAG) segundo raça/cor até a semana epidemiológica número 24. Pode-se verificar que a taxa de hospitalização por SRAG entre pacientes indígenas e amarelos permaneceram estáveis em todos os boletins epidemiológicos. Entre os indígenas, a menor taxa de hospitalização foi verificada nos boletins no 09 e no $14(0,2 \%)$ e maior no boletim no $18(0,42 \%)$. Já entre as pessoas de raça/cor amarela, a menor taxa foi verificada no boletim $n^{\circ} 18(1,48 \%)$ e maior taxa de hospitalização no boletim no 9 (2,9\%).

Por outro lado, as taxas de pessoas brancas e negras apresentaram divergências significativas. Pessoas brancas apresentaram declínio progressivo nas taxas de internação por SRAG em todos os boletins epidemiológicos estudados, reduzindo de $73 \%$ no boletim epidemiológico $n^{\circ} 9$ para $43,3 \%$ no boletim

Já entre pessoas negras, a taxa de internação por SRAG aumentou em todos os boletins epidemiológicos estudados, saindo de $23,9 \%$ no boletim epidemiológico nº 9 para $54,7 \%$ no boletim no 18 .

$n^{\circ} 18$. Já entre pessoas negras, a taxa de internação por SRAG aumentou em todos os boletins epidemiológicos estudados, saindo de $23,9 \%$ no boletim epidemiológico no 9 para $54,7 \%$ no boletim $n^{\circ} 18$.

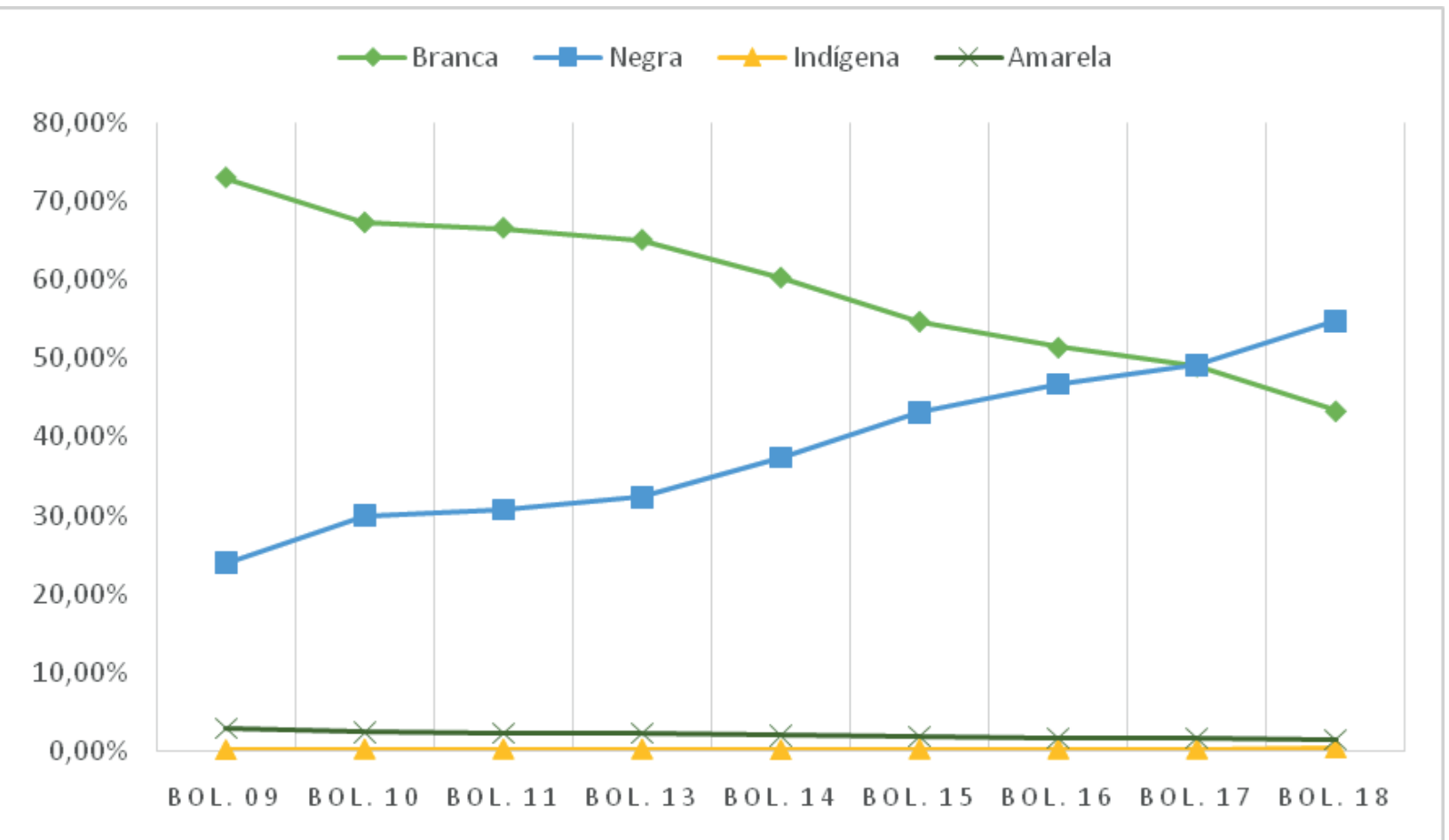

Gráfico 1. Evolução das taxas de hospitalização por Síndrome Respiratória Aguda Grave segundo raça/cor até 15 de junho de 2020.

Fonte: Dados dos boletins epidemiológicos coronavírus do Ministério da Saúde.

O gráfico 2 mostra a mortalidade por COVID-19 segundo a variável raça/cor até a semana epidemiológica número 24. Pode-se constatarque as taxas de óbito por COVID-19 entre pacientes indígenas e amarelos também permaneceram estáveis em todos os boletins epidemiológicos estudados. Entre os indígenas, a menor taxa óbito foi verificada nos boletins no 14 e $n^{\circ} 15(0,3 \%)$ e maior nos boletins $n^{\circ} 10, n^{\circ} 11, n^{\circ} 16, n^{\circ} 17$ e $n^{\circ} 18(0,5 \%)$. Já entre as pessoas de raça/cor 
amarela, a menor taxa de mortalidade foi verificada nos boletins $n^{\circ} 17$ e $n^{\circ} 18(1,6 \%)$ e maior no boletim no $11(2,7 \%)$.

Assim como nas taxas de internação por SRAG, a mortalidade por COVID-19 entre pessoas brancas e negras apresentaram divergências significativas. Pessoas de raça/cor branca apresentaram declínio progressivo nas taxas de óbito por COVID-19 em todos os boletins epidemiológicos publicados pelo

Já entre pessoas negras, a taxa de mortalidade por COVID-19 aumentou em todos os boletins epidemiológicos estudados, saindo de $34,3 \%$ no boletim epidemiológico nº 9 para $61,3 \%$ no boletim no 18. Ministério da Saúde, reduzindo de 62,9\% no boletim epidemiológico n॰ 9 para 36,5\% no boletim epidemiológico n 18. Já entre pessoas negras, a taxa de mortalidade por COVID-19 aumentou em todos os boletins epidemiológicos estudados, saindo de $34,3 \%$ no boletim epidemiológico n० 9 para $61,3 \%$ no boletim no 18 .

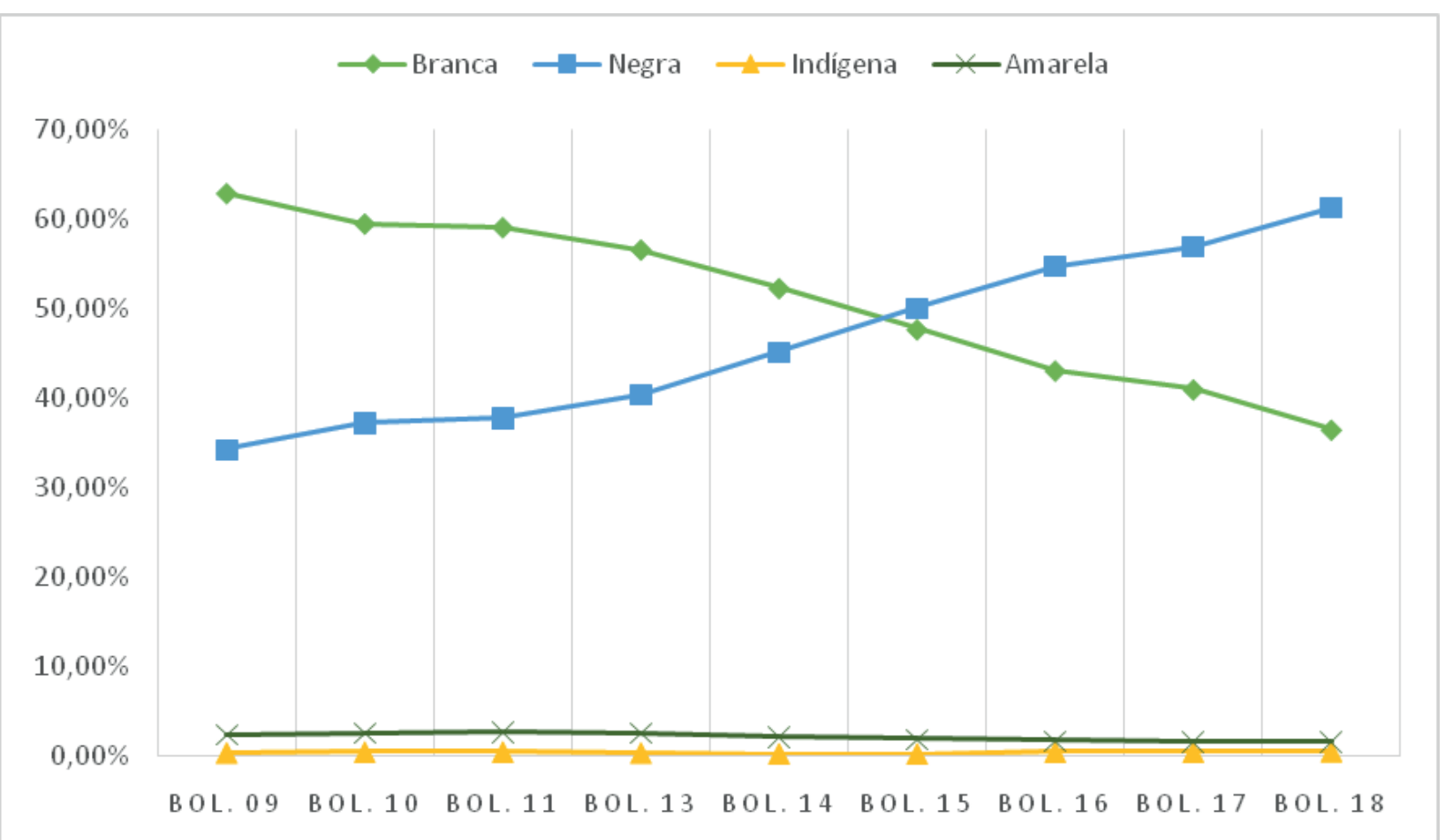

Gráfico 2. Evolução das taxas de óbito por COVID-19 segundo raça/cor até 15 de junho de 2020. Fonte: Dados dos boletins epidemiológicos coronavírus do Ministério da Saúde.

\section{Discussão}

A evoluçãoepidemiológica da pandemia por COVID-19 no Brasil aponta o aumento progressivo, tanto da mortalidade quanto das internações por SRAG entre pessoas negras, ao mesmo tempo em que revela queda sustentada nas mesmas taxas entre pessoas brancas. Percebe-se assim que, com a progressão da pandemia no país, a população negra tem se mostrado em maior situação de vulnerabilidade.

\begin{abstract}
A evolução epidemiológica da pandemia por COVID-19 no Brasil aponta o aumento progressivo, tanto da mortalidade quanto das internações por SRAG entre pessoas negras, ao mesmo tempo em que revela queda sustentada nas mesmas taxas entre pessoas brancas.
\end{abstract}

A inversão nas taxas de internação por SRAG entre brancos e negros ocorreu na semana epidemiológica 21 (17 a 23 de maio de 2020), enquanto a inversão nas taxas de mortalidade 
ocorreu de forma ainda mais precoce, na semana epidemiológica 19 (03 a 09 de maio). Tanto o aumento sustentado nas taxas de internação e óbito entre negros, quanto à redução constante na hospitalização e óbito entre pessoas brancas, nos faz constatar que pessoas negras têm maiores chances de internação por SRAG e morte por COVID-19 no Brasil. Tal constatação também foi evidenciada nos Estados Unidos, onde afro-americanos apresentaram piores desfechos da doença quando comparado à pessoas brancas ${ }^{20}$.

No que se refere à realidade brasileira, acredita-se que esse cenário desfavorável para pessoas de raça-cor negra pode ser explicado a partir de quatro fatores: a dificuldade ou impossibilidade de realização de isolamento social, à prevalência de comorbidades que precipitam quadros graves do COVID-19, as dificuldades no acesso aos serviços de saúde e ao racismo institucional.

\section{A problemática do isolamento social seletivo}

Por tratar-se de um vírus de alta capacidade de transmissão, o isolamento social agressivo, com realização de quarentena e fechamento de todos os serviços não-essenciais como escolas e locais de trabalho tem se mostrado, em diferentes partes do mundo, um instrumento importante para mitigar a transmissão da doença ${ }^{21}$. Entretanto, no cenário brasileiro, de forte desigualdade socioeconômica, manter-se em isolamento social é um privilégio que poucos têm acesso.

As favelas brasileiras, ocupadas majoritariamente por pessoas negras, são formadas por um conglomerado de casas e pessoas pobres de alta densidade demográfica, onde não há planejamento urbano, apresenta baixos índices de desenvolvimento humano, difícil e escasso acesso aos bens e serviços públicos, assim como ao saneamento básico e à educação sanitária, aspectos fundamentais para controle da doença ${ }^{22}$.

Isso não se resume apenas à realidade brasileira. Segundo Devakumare colaboradores ${ }^{23}$, apesar do vírus ter a capacidade de contaminar qualquer pessoa, a fragilidade socioeconômica e a baixa capacidade dos Estados em propor políticas específicas tem como consequência os piores desfechos do covid-19 às minorias étnico-raciais.

Além disso, para que as pessoas consigam se manter isoladas, é necessário que exista proteção social aos trabalhadores, uma vez que poucas formas de trabalho podem ser executadas de maneira remota. Em um estudo desenvolvido no Brasil, que avaliou o trabalho informal na perspectiva de gênero e raça, evidenciou que a população negra ocupa majoritariamente empregos informais e sem carteira assinada. Por outro lado, as pessoas brancas têm maior proteção ao emprego uma vez que, em maior proporção, possuem ocupação formal e com carteira assinada ${ }^{24}$.

Isso implica à população negra uma maior chance de desemprego durante a crise econômica gerada pela pandemia e dependência do Estado para garantir sua subsistência durante o período de isolamento social. Contudo, ao compreender o Estado brasileiro como uma estrutura que historicamente garantiu a manutenção do status quo, com pouca garantia de direitos, percebese que parcela significativa da população negra dificilmente conseguirá realizar o isolamento social, com garantia de subsistência.

Soma-se ainda o fato de a população negra ter menor acesso ao saneamento básico ${ }^{25}$. Neste sentido, ainda que consigam realizar o isolamento social mantendo-se em casa durante o período de pandemia, o fato de parcela significativa desta população não usufruir do saneamento básico, 
resulta em maior dificuldade em implementar medidas de higiene como a lavagem básica das mãos de forma periódica, o que também pode impactar em maior acometimento do sar-cov- 2 .

Nota-se, assim, que a situação de pobreza, a precária condição de moradia, a instabilidade no emprego e a alta prevalência de vínculos precários de trabalho, somados a falta de acesso ao saneamento básico resultam em maior dificuldade de pessoas negras realizarem o isolamento social e, consequentemente, maior chance de acometimento da doença.

\section{O perverso histórico de comorbidades na população negra}

Além das dificuldades relacionadas ao isolamento social, a população negra enfrenta alta prevalência de comorbidades. Estudo internacional com metanálise evidenciou que a presença de comorbidades como HAS, DM, doenças dos aparelhos respiratório e cardiovascular podem ser fatores de risco para o desenvolvimento de quadros graves do Covid-1926.

No Brasil, pessoas negras têm maior prevalência de HAS e DM do que pessoas brancas ${ }^{27,28}$. Além disso, em comunidades pobres e majoritariamente negra, é prevalente a existência de quadros de subnutrição infantil ou obesidade entre adultos, devido à falta de acesso a alimentos de boa qualidade, com controle de sódio e calorias ${ }^{22}$. Esta realidade impacta diretamente em maior prevalência de doenças cardiovasculares nesse grupo racial. Nesse aspecto, pode-se inferir que a população negra, ao possuir maior prevalência de comorbidades associadas ao quadro grave do Covid-19, tem maior possibilidade de óbito, quando comparada às pessoas brancas.

Entretanto, parcela significa dos estudos que abordam a prevalência de doenças em pessoas de raça-cor negra discutem os resultados a partir de uma análise de aspectos biológicos. Por outro lado, acreditamos que a alta prevalência de doenças crônicas também está atrelada ao racismo institucional, que tem como consequência a negligência, pior condição de assistência e a maior dificuldade de acesso aos serviços de saúde pelas pessoas negras, algo já reconhecido pela Organização Mundial de Saúde ${ }^{29}$.

Os dados brasileiros ainda são bastante incipientes, uma vez que há forte subnotificação relacionada ao preenchimento do quesito raça/cor dos mortos e infectados por coronavírus. Entretanto, os dados condensados nos Boletins Epidemiológicos do Ministério da Saúde mostram que, apesar da população branca ter sido mais acometida no início do surto no país, as manifestações graves da doença e a letalidade apresentam-se maiores em pessoas negras $^{11-19}$.

Esses dados mostram uma mudança no perfil de acometimento da doença, uma vez que no início da pandemia, a contaminação concentrou-se em pessoas brancas, de alto poder aquisitivo e que realizavam viagens internacionais para China e Europa. Porém, com o avanço da doença para as favelas e para municípios de pequeno porte, o percentual de acometimento da doença e letalidade tende a ser crescente na população negra.

\section{As dificuldades no acesso aos serviços de saúde}

Acredita-se que tanto a prevalência de comorbidades quanto a maior taxa de letalidade não podem ser explicadas exclusivamente por características genéticas e/ou biológicas. Parcela significativa da população negra não tem ou possui maiores dificuldades de acesso aos serviços de saúde devido à existência de racismo institucional ${ }^{30}$. 
Como categoria de análise, o acesso aos serviços de saúde foi amplamente discutido por Assis e Jesus $^{31}$. Trata-se de uma avaliação complexa e multidimensional que envolve questões políticas, econômico-sociais, organizacionais, técnicas e simbólicas.

Pela condição histórica de iniquidade às pessoas pretas, o racismo institucional influencia todas as dimensões do acesso aos serviços de saúde ${ }^{8}$. Não se pode esquecer que a história da população negra no Brasil é marcada por exploração e expropriação, que mesmo com a abolição formal da escravidão em 1888, a inexistência de uma reparação histórica impôs ao negro brasileiro menor acesso à educação e à informação ${ }^{32}$.

No contexto da pandemia, a baixa qualidade de vida das pessoas negras em geral, associada ao menor grau de escolaridade, pode resultar em maior dificuldade de identificação dos sinais e sintomas de gravidade da doença, fazendo com que esse público procure o serviço de saúde quando o quadro patológico encontra-se avançado, o que pode explicar, em partes, a progressão sustentada na letalidade evidenciada nos boletins epidemiológicos.

Acrescenta-se ainda que a pandemia possa provocar acesso limitado à assistência de outras comorbidades. Na Nigéria, um estudo apontou que a pandemia pode provocar efeitos de curto e longo prazo no acometimento a outras doenças como o HIV. Dentre os impactos possíveis está o acesso limitado a medicamentos, que resultará em maior resistência às drogas, redução de acompanhamento médico e maior incidência da doença ${ }^{33}$.

No Brasil, além das doenças já mencionadas, pessoas negras também estão em maior situação de vulnerabilidade para o acometimento do HIV 34,35 . Esses fatores, somado a maior dependência do sistema público de saúde e a intensificação do subfinaciamento do SUS pela Emenda Constitucional 95/2016 ${ }^{36}$, apontam para piores condições de acesso aos serviços de saúde, tanto para o coronavírus quanto para a assistência das doenças prevalentes nesse grupo racial.

\section{Conclusão}

O estudo evidenciou o aumento sustentado nas taxas de internação e óbito entre pessoas de raça/cor negra e a redução constante na hospitalização e óbito entre pessoas brancas. Dessa forma, constata-se que pessoas negras têm maiores chances de internação por SRAG e morte por COVID-19 no Brasil, o que implica maior situação de vulnerabilidade às pessoas desse grupo racial. Acredita-se que esse cenário desfavorável entre pessoas negras se deve a dificuldade ou impossibilidade de realização de isolamento social, à prevalência de comorbidades que precipitam quadros graves do COVID-19, às dificuldades no acesso aos serviços de saúde e ao racismo institucional.

Com isso, faz-se necessário a implementação de uma rede de proteção social a esse grupo racial, com o objetivo de reduzir o acometimento da doença e a letalidade do vírus. Esse enfrentamento depende da maior disponibilidade de recursos e articulação de setores governamentais com grupos organizados da sociedade civil para o fortalecimento da Política Nacional de Saúde Integral da População Negra. Acredita-se que as ações devam abarcar os mais diversos setores: social, econômico e assistencial.

As ações nas comunidades devem ser guiadas através da avaliação dos territórios, com identificação dos grupos de risco, orientação acerca das medidas de higiene, oferta de álcool em gel a 70\% para as famílias com acesso escasso a água e garantia de espaços para realização de quarentena dos casos confirmados. Além disso, é fundamental que o Estado garanta que as 
pessoas negras possam realizar o isolamento social com aporte financeiro para subsistência e implementação de políticas de proteção ao emprego.

Além disso, é necessário capacitar os profissionais de saúde, tanto da atenção básica quanto da rede hospitalar para atenção à população negra, na tentativa de controlar a disseminação da doença e avaliação precoce das pessoas com maior potencial para evolução para o quadro grave da doença.

Conflito de interesses: Não se aplica.

Financiamento: Não se aplica.

\section{Referências}

1. World Health Organization. Coronavirus disease (COVID-19) outbreak. https://www.who.int/emergencies/diseases/novel-coronavirus-2019

2. Brasil. Ministério da Saúde. Painel Coronavírus, 2020. https://covid.saude.gov.br/_

3. Fundação Oswaldo Cruz. Nota Técnica - Covid-19 E Vulnerbilidades: Considerações Sobre Proteção Social Nas Favelas. Observatório COVID-19, 2020. https://portal.fiocruz.br/ documento/nota-tecnica-covid-19-e-vulnerabilidades-consideracoes-sobre-protecaosocial-nas-favelas

4. Carrapato P, Correia P, Garcia B. Determinante da saúde no Brasil: a procura da equidade na saúde. Saúde soc. 2017;26(3):676-689. https://doi.org/10.1590/s0104-12902017170304

5. Theophilo RL, Rattner D, Pereira EL. Vulnerabilidade de mulheres negras na atenção ao pré-natal e ao parto no SUS: análise da pesquisa da Ouvidoria Ativa. Ciênc. saúde coletiva. 2018;23(11):3505-3516. https://doi.org/10.1590/1413-812320182311.31552016

6. Alio AP, Richman AR, Clayton HB, Jeffrs DF, Wathington DJ, Salihu HM. An ecological approach to understanding black-white disparities in perinatal mortality. Matern Child Health J. 2010;14(4):557-66. https://doi.org/10.1007/s10995-009-0495-9

7. Lawrence HP, Cidro J, Isaac-Mann S, Peressini S, Maar M, Schroh RJ, et al. Racism and Oral Health Outcomes among Pregnant Canadian Aboriginal Women. J Health Care Poor Underserved. 2016;27(1 Suppl):178-206._https://doi.org/10.1353/hpu.2016.0030

8. Werneck J. Racismo institucional e saúde da população negra. Saude soc. 2016; 25(3):535549. https://doi.org/10.1590/s0104-129020162610

9. Umeukeje EM, Wild MG, Maripuri S, Davidson T, Rutherford M, Abdel-Kader K, et al. Black American 'Perspectives of Barreiras and Facilitators of Community Screening for Kidney Disease. Clin J Am Soc Nephrol. 2018;13(4):551-559. https://doi.org/10.2215/CJN.07580717

10.Arriola KJ. Race, Racism, and Access to Renal Transplantation among African Americans. J Health Care Poor Underserved. 2017;28(1):30-45. https://doi.org/10.1353/hpu.2017.0005

11.BRASIL. Ministério da Saúde. Secretaria de Vigilância em Saúde. Boletim Epidemiológico 09 - Situação Epidemiológica da Covid-19: doença pelo novo coronavírus 2019. Brasília: Ministério da Saúde, 2020;09:1-15. Disponível em: https://portalarquivos.saude.gov.br/ images/pdf/2020/April/12/2020-04-11-BE9-Boletim-do-COE.pdf

12.BRASIL. Ministério da Saúde. Secretaria de Vigilância em Saúde. Boletim Epidemiológico 10 - Situação Epidemiológica da Covid-19: doença pelo coronavírus 2019. Brasília: Ministério da Saúde, 2020;10:1-31. Disponível em: https://portalarquivos.saude.gov.br/images/ pdf/2020/April/17/2020-04-16---BE10---Boletim-do-COE-21h.pdf

13.BRASIL. Ministério da Saúde. Secretaria de Vigilância em Saúde. Boletim Epidemiológico 11: doença pelo novo coronavírus 2019 (COVID-19). Brasília: Ministério da Saúde, 2020;11:137. Disponível em: https://portalarquivos.saude.gov.br/images/pdf/2020/April/18/2020-0417---BE11---Boletim-do-COE-21h.pdf 
14.BRASIL. Ministério da Saúde. Secretaria de Vigilância em Saúde. Boletim COE COVID-19 número 13 - Situação epidemiológica: doença pelo novo coronavírus 2019. Brasília: Ministério da Saúde, 2020;13:1-18.

Disponível em: https://coronavirus.saude.gov.br/boletins-epidemiologicos

15.BRASIL. Ministério da Saúde. Secretaria de Vigilância em Saúde. Boletim Epidemiológico Especial COE-COVID19 número 14. Brasília: Ministério da Saúde, 2020;14:1-38.

Disponível em: https://portalarquivos.saude.gov.br/images/pdf/2020/April/27/2020-04-2718-05h-BEE14-Boletim-do-COE.pdf

16.BRASIL. Ministério da Saúde. Secretaria de Vigilância em Saúde. Boletim Epidemiológico Especial COE-COVID19 número 15. Brasília: Ministério da Saúde, 2020;15:1-68.

Disponível em: https://portalarquivos.saude.gov.br/images/pdf/2020/May/09/2020-05-06BEE15-Boletim-do-COE.pdf

17.BRASIL. Ministério da Saúde. Secretaria de Vigilância em Saúde. Boletim Epidemiológico Especial COE-COVID19 número 16. Brasília: Ministério da Saúde, 2020;16:1-67.

Disponível em: https://portalarquivos.saude.gov.br/images/pdf/2020/May/21/2020-05-19--BEE16---Boletim-do-COE-13h.pdf

18.BRASIL. Ministério da Saúde. Secretaria de Vigilância em Saúde. Boletim Epidemiológico Especial COE-COVID19 número 17. Brasília: Ministério da Saúde, 2020;17:1-74.

Disponível em: https://www.saude.gov.br/images/pdf/2020/May/29/2020-05-25---BEE17--Boletim-do-COE.pdf

19.BRASIL. Ministério da Saúde. Secretaria de Vigilância em Saúde. Boletim Epidemiológico Especial doença pelo coronavírus número 18. Brasília: Ministério da Saúde, 2020;18:1-42. Disponível em: http://saude.gov.br/images/pdf/2020/June/18/Boletim-epidemiologicoCOVID-2.pdf

20.Ferdinand KC, Nasser SA. African-American COVID-19 Mortality: A Sentinel Event. J Am Coll Cardiol. 2020;75(21):2746-2748. https://doi.org/10.1016/j.jacc.2020.04.040

21.Koo JR, Cook AR, Park M, Sun Y, Sun H, Lim JT, et al. Interventions to mitigate early spread of SARS-CoV-2 in Singapore: a modelling study. Lancet Infect Dis. 2020;20:678-88. https://doi.org/10.1016/S1473-3099(20)30162-6.

22.Sawaya AL; Albuquerque MP, Domene SMA. Violência em favelas e saúde. Estud. av., 2018; 32(93):243-250. https://doi.org/10.5935/0103-4014.20180041.

23.Devakumar D, Shannon G, Bhopal S, Abubakar I. Racism and discrimination in COVID-19 responses. Lancet. 2020;395(10231):1194. https://doi.org/10.1016/S0140-6736(20)30792-3

24.Araujo AMC, Lombardi MR. Trabalho informal, gênero e raça no Brasil, no início do século XXI. Cad. Pesqui. 2013;43(149):452-477. https://doi.org/10.1590/S0100-15742013000200005

25.Brasil. Ministério da Saúde. Secretaria de Gestão Estratégica e Participativa. Painel de Indicadores do SUS - Temático Saúde da População Negra. Brasília: Ministério da Saúde, 2016;7(10). https://pesquisa.bvsalud.org/bvsms/resource/pt/mis-38998

26.Yang J, Zheng Y, Gou X, Pu K, Chen Z, Guo Q, et al. Prevalence of comorbidities and its effects in patients infected with SARS-CoV-2: a systematic review and meta-analysis. Int J of Infect Dis. 2020;94:91-95. 1 https://doi.org/10.1016/j.ijid.2020.03.017.

27.Andrade SSA, Stopa SR, Brito AS, Chueri PS, Szwarcwald CL, Malta DC. Prevalência de hipertensão arterial autorreferida na população brasileira: análise da Pesquisa Nacional de Saúde, 2013. Epidemiol. Serv. Saúde. 2015;24(2):297-304.

https://doi.org/10.5123/S1679-49742015000200012

28.Malta DC, Ducan BB, Schmidt MI, Machado IE, Silva AG, Bernal RTI. Prevalência de diabetes mellitus determinada pela hemoglobina glicada na população adulta brasileira, Pesquisa Nacional de Saúde. Rev. bras. epidemiol. 2019;22(Suppl 2):E190006.SUPL.2.

https://doi.org/10.1590/1980-549720190006.supl.2 
29.ORGANIZACIÓN MUNDIAL DE LA SALUD. Salud y ausencia de discriminación. Ginebra, 2001. Documento de la OMS para la Conferencia Mundial Contra el Racismo, la Discriminación Racial, la Xenofobia y las Formas Conexas de Intolerancia. (Serie de publicaciones sobre salud y derechos humanos, 2).

30.Silva NN, Favacho VBC, Boska GA, Andrade EC, Merces NP, Oliveira MAF. Acesso da população negra a serviços de saúde: revisão integrativa. Rev. Bras. Enferm. 2020;73 (4):e20180834. https://doi.org/10.1590/0034-7167-2018-0834.

31.Assis MMA, Jesus WLA. Acesso aos serviços de saúde: abordagens, conceitos, políticas e modelo de análise. Ciênc. saúde col. 2012;17(11):2865-2875. http://dx.doi.org/10.1590/S1413-81232012001100002.

32. Thiede $\mathbf{M}$, Mcintyre $\mathbf{D}$. Information, communication and equitable access to health care: a conceptual note. Cad. Saúde Pública. 2008;24(5):1168-1173. https://doi.org/10.1590/S0102-311X2008000500025

33.Oladele TT, Olakunde BO, Oladele EA, Ogbuoji O, Yamey G. The impact of COVID-19 on HIV financing in Nigeria: a call for proactive measures. BMJ Global Health. 2020;5:e002718. https://doi.org/10.1136/bmjgh-2020-002718

34.Andrade JS, Lima GS, Oliveira FBM. Analysis of the vulnerability factors to HIV / AIDS infection in the black population. Re on Facema. 2018;4(3):986-992.

35.Taquette SR, Meirelles ZV. Discriminação racial e vulnerabilidade às DST/Aids: um estudo com adolescentes negras. Physis. 2013;23(1):129-142. https://doi.org/10.1590/S0103-73312013000100008

36.Mariano CM. Emenda constitucional 95/2016 e o teto dos gastos públicos: Brasil de volta ao estado de exceção econômico e ao capitalismo do desastre. Rev. Investig. Const. 2017;4(1):259281. http://dx.doi.org/10.5380/rinc.v4i1.50289 